\title{
Low-voltage magnetoresistance in silicon
}

\author{
ARISING FRom C. H. Wan, X. Z. Zhang, X. L. Gao, J. M. Wang \& X. Y. Tan Nature 477, 304-307 (2011)
}

Magnetoresistance exhibited by non-magnetic semiconductors has attracted much attention ${ }^{1-13}$. In particular, Wan et al. reported roomtemperature magnetoresistance in silicon to reach $10 \%$ at $0.07 \mathrm{~T}$ and $150,000 \%$ at $7 \mathrm{~T}$ - "an intrinsically spatial effect" 12 . Their supply voltage was approximately $10 \mathrm{~V}$ ( ref. 12), which is low and approaches the industrial requirement ${ }^{14}$. However, we have found their large magnetoresistance values to be experimental artefacts caused by their method of measurement. The true room-temperature magnetoresistance of the devices described in ref. 12 is low with a magnetic field of up to $7 \mathrm{~T}$ and a supply voltage of around $10 \mathrm{~V}$ and hence these devices cannot offer large magnetoresistance with low supply voltage to industry. There is a Reply to this Brief Communication Arising by Zhang, X. Z., Wan, C. H., Gao, X. L., Wang, J. M. \& Tan, X. Y. Nature 501, http://dx.doi.org/ 10.1038 /nature12590 (2013).

Wan et al. ${ }^{12}$ measured two types of $\mathrm{In} / \mathrm{SiO}_{2} / \mathrm{Si} / \mathrm{SiO}_{2} / \mathrm{In}$ devices using a Keithley 2400 sourcemeter as both a current source and a voltage meter (which we refer to here as method 1), and obtained large magnetoresistance values of up to $10 \%$ at $0.07 \mathrm{~T}$ and $150,000 \%$ at $7 \mathrm{~T}$. We fabricated two devices with the same structures as those of ref. 12 and performed method 1 using them. Their voltage-current $(V-I)$ curves can be divided into different regions with different resistances, just as in the results of ref. 12 . Wan et al. ${ }^{12}$ claim that injection of minority carriers into silicon causes a $\mathrm{p}-\mathrm{n}$ junction and the changes in resistance, that large magnetoresistance occurs with applied current in one of the regions (referred as to the transition region), and that the magnetic-field dependence of the magnetoresistance in the transition region is different from those in the other regions. However, when we used another method (here called method 2) with unchanged measuring parameters and different instruments on the devices, the $V-I$ characteristics without the transition region were obtained. The only difference between the two methods is that in method 2 we used the Keithley 2400 only as the current source, with an independent voltmeter (Keithley 2182) as the voltage meter.

Further, we performed both methods on two circuits composed of linear resistors, which were used to simulate the devices. The results indicate that in method 1 the Keithley 2400 itself interferes with the measurement of the specimen and cannot give correct voltage values when the applied current exceeds a certain value and falls in the transition region. Because ref. 12 claims that large magnetoresistances were measured when $I$ was in the transition region, magnetoresistance was defined as $[R(B)-R(B=0)] / R(B=0)$ and $R=V / I$, we conclude that the large magnetoresistance values are really experimental artefacts caused by the interference of the sourcemeter. Method 2 is valid. Using it, we obtained magnetoresistance values for the two devices with supply voltages of $6.7-72 \mathrm{~V}$ and $0.79-50 \mathrm{~V}$, respectively. The values are all low and the magnetic-field dependence at all applied currents is the same (above $2 \mathrm{~T}$ the field dependence is linear); the magnetoresistance does not exhibit any signs of saturation at fields up to $7 \mathrm{~T}$. The linear dependence without magnetoresistance saturation is the same as for inhomogeneity-induced magnetoresistance ${ }^{7-9}$.

\section{Jun Luo ${ }^{1}$, Peisen $\mathrm{Li}^{2}$, Sen Zhang ${ }^{2}$, Hongyu Sun ${ }^{1}$, Hongping Yang ${ }^{1}$ \& Yonggang Zhao ${ }^{2}$ \\ ${ }^{1}$ Beijing National Center for Electron Microscopy, Laboratory of Advanced Materials, Department of Materials Science and Engineering, Tsinghua University, Beijing 100084, China. \\ email: jluo@mail.tsinghua.edu.cn \\ ${ }^{2}$ Department of Physics and State Key Laboratory of Low-Dimensional Quantum Physics, Tsinghua University, Beijing 100084, China.}

Received 20 September 2012; accepted 16 August 2013.

1. Smith, R. A. Semiconductors 2nd edn, Ch. 5 (Cambridge Univ. Press, 1978).

2. Popović, R. S. Hall Effect Devices Ch. 3 (IOP Publishing, 1991).

3. Xu, R. etal. Large magnetoresistance in non-magnetic silver chalcogenides. Nature 390, 57-60 (1997).

4. Solin, S. A., Thio, T., Hines, D. R. \& Heremans, J. J. Enhanced room-temperature geometric magnetoresistance in inhomogeneous narrow-gap semiconductors. Science 289, 1530-1532 (2000).

5. Lee, M., Rosenbaum, T. F., Saboungi, M.-L. \& Schnyders, H. S. Band-gap tuning and linear magnetoresistance in the silver chalcogenides. Phys. Rev. Lett. 88, 066602 (2002).

6. Husmann, A. et al. Megagauss sensors. Nature 417, 421-424 (2002).

7. Parish, M. M. \& Littlewood, P. B. Non-saturating magnetoresistance in heavily disordered semiconductors. Nature 426, 162-165 (2003).

8. Hu, J. S. \& Rosenbaum, T. F. Classical and quantum routes to linear magnetoresistance. Nature Mater. 7, 697-700 (2008).

9. Delmo, M. P., Yamamoto, S., Kasai, S., Ono, T. \& Kobayashi, K. Large positive magnetoresistive effect in silicon induced by the space-charge effect. Nature 457, 1112-1115 (2009).

10. Delmo, M. P., Kasai, S., Kobayashi, K. \& Ono, T. Current-controlled magnetoresistance in silicon in non-Ohmic transport regimes. Appl. Phys. Lett. 95, 132106 (2009).

11. Schoonus, J. J. H. M., Haazen, P. P. J., Swagten, H. J. M. \& Koopmans, B. Unravelling the mechanism of large room-temperature magnetoresistance in silicon. J. Phys. D 42, 185011 (2009).

12. Wan, C. H., Zhang, X. Z., Gao, X. L., Wang, J. M. \& Tan, X. Y. Geometrical enhancement of low-field magnetoresistance in silicon. Nature 477, 304-307 (2011).

13. Porter, N. A. \& Marrows, C. H. Linear magnetoresistance in $n$-type silicon due to doping density fluctuations. Sci. Rep. 2, 565 (2012).

14. Allan, A. et al. 2011 edn, http://www.itrs.net/Links/2011ITRS/Home2011.htm (2011).

Author Contributions J.L. designed the research blueprint, performed the experiments and data analysis, and wrote the manuscript. P.L. and S.Z. assisted in the magnetoresistance measurement and data analysis. H.S. and H.Y. assisted in the data collection. Y.Z. supervised the magnetoresistance measurement and contributed to the data analysis and manuscript writing.

Competing Financial Interests Declared none.

doi: $10.1038 /$ nature 12589

\section{Zhang et al. reply}

REPLYING To J. Luo et al. Nature 501, http://dx.doi.org/10.1038/nature12589 (2013)

We agree with Luo et al. ${ }^{1}$ that the magnetoresistance effects that we reported ${ }^{2}$ were dependent on the method used to measure them. The reason that there is a difference in the results depending on whether method 1 or method 2 is used (adopting the measurement notation of ref. 1) is that there are two voltage-stabilizing diodes in the Keithley 2400 instrument we used. We were unaware that when this instrument was used both as current source and voltmeter, one diode connected the input port of the current source to the input port of the 
voltmeter, whereas the other diode connected the output port of the current source to the output port of the voltmeter. The diodes caused a crossover of the Hall coefficient from negative to positive when the instrument was used to conduct a Hall measurement in this configuration, leading us to propose an invalid mechanism for the abnormal magnetoresistance. Therefore the mechanism we proposed ${ }^{2}-$ minority injection and an induced p-n boundary-does not provide a correct explanation for the observed geometry-enhanced magnetoresistance. Although such a mechanism does not operate in our samples, we note that a $\mathrm{p}-\mathrm{n}$ boundary could still enhance magnetoresistance in certain circumstances according to our and others' theoretical calculations and experiments ${ }^{2-4}$.

Once we became aware of the existence and role of the diodes, we were able to show that all the magnetoresistance properties reported previously ${ }^{2}$ could be qualitatively reproduced by integrating two diodes between the indium electrodes within the devices themselves, resulting in magnetoresistance devices - consisting of the silicon wafer, the indium electrodes and now also the diodes-whose apparent properties are no longer dependent on the measurement methodology.

We are now developing a new mechanistic framework to describe this phenomenon, which takes into account the influences of both device geometry and the newly incorporated diodes. The mechanism and its generalization to other materials, (accompanied by corrections to our earlier invalid explanation) will be described in a forthcoming paper.

Finally, it is worth noting that (1) this magnetoresistance device could still be integrated into silicon-based electronics and therefore aid the development of magnetoelectronics, (2) the new mechanism potentially provides ways to optimize device performance, such as improving the low-field sensitivity and reducing the power required and (3) our research ${ }^{2}$ shows that the low-field magnetoresistance of $(\mu B)^{2}$ restricted by the Onsager relation ${ }^{5}$ could be enhanced in the nonlinear transport region, suggesting a way to improve the low-field sensitivity of semiconductors with only moderate mobility.

We thank Yang Ji at the Chinese Academy of Sciences, who initially alerted us to the methodology-dependence of our original results.

X. Z. Zhang ${ }^{1,2}$, C. H. Wan ${ }^{1,2}$, X. L. Gao ${ }^{1,2}$, J. M. Wang ${ }^{1,2}$ \& X. Y. Tan ${ }^{1,2}$

${ }^{1}$ Laboratory of Advanced Materials, Department of Materials Science and Engineering, Tsinghua University, Beijing 100084, China.

${ }^{2}$ Beijing National Center for Electron Microscopy, Tsinghua University, Beijing 100084, China.

email: xzzhang@tsinghua.edu.cn

1. Luo, J. et al. Low-voltage magnetoresistance in silicon. Nature $\mathbf{5 0 1}$, http://dx.doi.org/10.1038/nature12589 (2013).

2. Wan, C. H., Zhang, X. Z., Gao, X. L., Wang, J. M. \& Tan, X. Y. Geometrical enhancement of low-field magnetoresistance in silicon. Nature 477, 304-307 (2011).

3. Hu, J. S. \& Rosenbaum, T. F. Classical and quantum routes to linear magnetoresistance. Nature Mater. 7, 697-700 (2008).

4. Parish, M. M. \& Littlewood, P. B. Non-saturating magnetoresistance in heavily disordered semiconductors. Nature 426, 162-165 (2003).

5. Baker, D. R. \& Heremans, J. P. Linear geometrical magnetoresistance effect: Influence of geometry and material composition. Phys. Rev. B 59, 13927-13942 (1999)

doi: $10.1038 /$ nature 12590 